\title{
Recombination rate coefficients of Be-like neon
}

\author{
I. Orban ${ }^{1}$, S. Böhm ${ }^{1}$, S. D. Loch $^{2}$, and R. Schuch ${ }^{1}$ \\ 1 Department of Atomic Physics, Stockholm University, 10691 Stockholm, Sweden \\ e-mail: istvan. orban@physto.se \\ 2 Department of Physics, Auburn University, Auburn, 36849 Alabama, USA
}

Received 30 May 2008 / Accepted 31 July 2008

ABSTRACT

\begin{abstract}
Aims. Merged-beam and plasma recombination rate coefficients for Be-like Ne VII were extracted from results of a merged-beam type experiment.

Methods. The CRYRING heavy-ion storage ring was used to determine merged-beam recombination rate coefficients for Be-like $\mathrm{Ne}^{6+}$. Recombined $\mathrm{Ne}^{5+}$ ions were separated from the stored beam in the first dipole magnet following the electron-ion interaction region. Field-ionization at this dipole magnet prevented detection of recombination into states with the principal quantum number $n>n_{\text {cutoff }}=$ 23. To account for the field-ionization effects, results obtained with AUTOSTRUCTURE calculations were used for recombination channels above $n_{\text {cutoff }}$. The merged-beam recombination rate coefficients were then convoluted with Maxwellian electron energy distributions in the $10^{3}-3 \times 10^{6} \mathrm{~K}$ temperature region, to obtain plasma recombination rate coefficients.

Results. Good agreement was found between the experimentally derived rate coefficient spectrum and results of the AUTOSTRUCTURE calculation. At low energies, several strong dielectronic recombination resonances belonging to the spin-forbidden $2 \mathrm{~s} 2 \mathrm{p}\left({ }^{3} \mathrm{P}_{\mathrm{J}}\right) n l$ series dominate the merged-beam spectrum. Recombination through these states dominates at low-temperatures, e.g. at $10^{3} \mathrm{~K}$ recombination through these resonances is more than one order of magnitude higher than the radiative recombination rate coefficient. Most data from the literature significantly underestimate the low-temperature plasma rate coefficients below $10^{4} \mathrm{~K}$, with only two calculations showing rate coefficients comparable to our results.

Strong contributions from trielectronic recombination were found in the merged-beam spectrum of Be-like Ne, associated with double excitation of the Be-like Ne core, during the attachment of the free electron. Calculated trielectronic recombination resonance positions agree with experimental peaks, however compared to the experiment, the calculation underestimates the strength of trielectronic recombination.
\end{abstract}

Key words. atomic data - atomic processes - line: formation - plasmas - Sun: general

\section{Introduction}

Dielectronic recombination (DR) is a resonant two step process, in which an electron recombines with a non-bare ion. In the first step of DR, an incoming electron is attached to the ion, with the simultaneous excitation of the core. The created doubly-excited state decays either by autoionization or by radiative decay. The autoionization channel returns the system to the original charge state, whereas if radiative decay leads to a bound state, DR is finalized and the charge of the ion decreases by one.

DR was first recognized by Burgess (1964) as being an important recombination mechanism in plasmas, dominating over the direct radiative recombination (RR) channel at high temperatures. Recombination rate coefficients of highly charged ions are relevant for the interpretation of astrophysical observations, for the study and modeling of astrophysical and laboratory plasmas. Neon is an astrophysically abundant element(Anders \& Grevesse 1989), and because of its inert properties, is often used as carrier gas in laboratory experiments. Reliable dielectronic recombination plasma rate coefficients are required for ionization balance calculations, extraction of elemental abundances from astrophysical observations and also for the calculation of radiative energy loss in optically thin plasmas.

Most DR data available in the literature originate from calculations. The energy position of DR into low- $n$ states or into states where the two participating electrons are strongly correlated is difficult to calculate. Often, calculations have large uncertainties in the low-energy DR resonance positions and consequently states that should be autoionizing can be calculated as being bound leading to no DR contribution from these resonances and an underestimation of the total DR rate coefficient. In a similar manner states that should be bound can be calculated as being autoionizing. The difficulty in calculating these low energy resonances can lead to low-temperature plasma rate coefficients having large uncertainties. Even small changes in the position of low-energy DR resonances result in large uncertainties of plasma rate coefficients, especially at temperatures where the corresponding ions are abundant in photoionized plasma (Schippers et al. 2004). Storage ring measurements are essential for determining accurate low-energy recombination rate coefficients and can benchmark different theoretical descriptions.

In Be-like ions, trielectronic recombination (TR) provides another important recombining channel, in addition to $\mathrm{RR}$ and DR. In the first step of TR, instead of the excitation of one core electron as in DR, two core electrons are excited to higher energies, and as a result a triply-excited state is formed. In Be-like ions, this first step is associated with the excitation of the $2 \mathrm{~s}^{2}$ core to a $2 \mathrm{p}^{2}$ configuration, with the simultaneous recombination of a free electron to a certain $n l$ Rydberg state. If the newly formed ion decays by radiative transition below its first ionization threshold, autoionization will no longer be possible, and trielectronic recombination is finalized. Because of the strong electron-electron interaction in the triply excited state, TR offers further information on electron correlation and it yields emission lines with specific energies. 
In storage ring experiments, TR was first observed to have important contributions in the recombination spectrum of Be-like Cl (Schnell et al. 2003). Later, Schippers et al. (2004) investigated the recombination spectrum of Be-like $\mathrm{Mg}$. For this ion distinct contribution from TR in the form of several sharp peaks was also found. Toward higher $\mathrm{Z}$ ions, some TR resonance features appear in the recombination spectrum of $\mathrm{Ti}^{18+}$ (Schippers et al. 2007) whereas in case of $\mathrm{Fe}^{22+}$ only one clear peak could be attributed to TR (Savin et al. 2006). Important TR contributions were observed by Fogle et al. (2005), in the spectra of Be-like $\mathrm{N}$ and Be-like O, whereas in the spectrum of Be-like $\mathrm{C}$, TR did not have a notable contribution. In case of Be-like $\mathrm{O}$, a low-energy TR resonance is almost entirely responsible for the plasma recombination rate coefficients below $10^{3} \mathrm{~K}$, and it solely shifts down the temperature where RR becomes the dominant recombination channel from $2250 \mathrm{~K}$ to $125 \mathrm{~K}$.

Here we report a first measurement of the recombination spectrum of Be-like $\mathrm{Ne}$ recombining into B-like Ne. The paper is structured as follows. The experimental procedure and the data analysis are presented in Sect. 2. The theoretical description is contained in Sect. 3. Our results are presented in Sect. 4, followed by comparison with data available in the literature, in Sect. 5. Conclusions and most important results are summarized at the end of the paper.

\section{Experiment and data analysis}

The recombination measurement was performed at the CRYRING heavy-ion storage ring(Abrahamsson et al. 1993) of the Manne Siegbahn Laboratory, in Stockholm. Be-like ${ }^{20} \mathrm{Ne}^{6+}$ ions were produced in an ECR ion source and were transported and injected in the storage ring. Following acceleration by an RF drift tube system, up to an energy of $\sim 8.36 \mathrm{MeV} /$ nucleon, an average of $10^{7}$ ions were orbiting in the storage ring. In the electron cooler (Danared et al. 2000) section of the storage ring, an electron beam with anisotropic velocity distribution, characterized by a longitudinal temperature of $k T_{\|}=0.2 \mathrm{meV}$ and a transversal temperature of $k T_{\perp}=1 \mathrm{meV}$, was merged with the circulating ion beam, and the ions were electron cooled (Poth 1990) for $1.5 \mathrm{~s}$. The electron-ion interaction length extended over $0.8 \mathrm{~m}$ and the electron density at cooling was $n_{\mathrm{e}}=3.8 \times 10^{6} \mathrm{~cm}^{-3}$.

Recombined B-like $\mathrm{Ne}^{5+}$ ions were separated from the stored beam in the first dipole magnet after the interaction region and were detected with $100 \%$ efficiency using a surface barrier detector. For every ion hiting the detector, the electron acceleration potential, pulse height and cycle time were recorded.

Following electron cooling, the electron-ion collision energy was scanned over a range covering all DR resonances associated with the excitation of a core electron within the same shell, hereafter termed $\Delta n=0$ type DR. During an acquisition timewindow of $\sim 8 \mathrm{~s}$, the same collision energy range was covered first with electrons faster, then with electrons slower than the orbiting ions. After completing the energy scan, the acquisition window was closed and the ion beam was dumped. A subsequent injection then started a new CRYRING machine-cycle.

After the measurement described above, the energy range was extended up to $200 \mathrm{eV}$, to cover DR resonances associated with excitation of a $2 \mathrm{~s}$ electron to higher $n$ shells. This spectrum was measured only with electrons faster than the ions.

Ions recombining into states with the outer electron having a principal quantum number $n>n_{\text {cutoff }}=23$ were field-ionized at the charge selecting dipole magnet. By returning to the original charge-state, the field-ionized ions were not separated from the stored beam and were lost for detection. Spectra containing recombination into states up to $n=23$ is hereafter designated as $n_{\text {cutoff }}$ spectra.

To obtain recombination rate coefficients, the count rate $R(E)$ associated with every electron-ion collision energy value was normalized to the number of ions $N_{\mathrm{i}}$ and electron density $n_{\mathrm{e}}$

$\alpha(E)=\frac{R(E) \gamma^{2}}{N_{\mathrm{i}} n_{\mathrm{e}}\left(l_{\mathrm{i}} / L_{\mathrm{R}}\right)}$,

where $\gamma$ is the relativistic factor. The time fraction spent by the ions in the interaction region was accounted for by the fraction $l_{\mathrm{i}} / L_{\mathrm{R}}$, where $l_{\mathrm{i}}$ and $L_{\mathrm{R}}$ are the electron-ion interaction length and ion orbit length, respectively. The measured spectrum was corrected for the $33 \mathrm{~s}$ lifetime of the ion beam and for the background signal, arising mostly from electron capture from residual gas molecules.

The electron-ion collision energy in the center-of-mass system was calculated using equation:

$$
\begin{aligned}
& E=\left[\left(E_{\mathrm{i}}+E_{\mathrm{e}}+M_{\mathrm{i}} c^{2}+m_{\mathrm{e}} c^{2}\right)^{2}\right. \\
& \left.-\left(\sqrt{E_{\mathrm{i}}^{2}+2 M_{\mathrm{i}} c^{2} E_{\mathrm{i}}}+\sqrt{E_{\mathrm{e}}^{2}+2 m_{\mathrm{e}} c^{2} E_{\mathrm{e}}}\right)^{2}\right]^{1 / 2}-M_{\mathrm{i}} c^{2}-m_{\mathrm{e}} c^{2}
\end{aligned}
$$

where $E_{\mathrm{i}}$ is the drag force corrected ion energy, $E_{\mathrm{e}}$ is the space charge corrected electron energy, $M_{\mathrm{i}}$ and $m_{\mathrm{e}}$ are the ion and electron mas, respectively, and $c$ is the speed of light.

Experimental uncertainties have been discussed in detail elsewhere (Orban et al. 2007). For the recombination spectrum of Be-like $\mathrm{Ne}$ we estimate uncertainties in the energy scale of up to a few percent.

\section{Theory}

Recombination cross sections were obtained from intermediatecoupling AUTOSTRUCTURE calculations (Badnell 1986, 1987), extending over the energy range corresponding to $\Delta n=0$ and $\Delta n=1$ type of DR. The calculations were performed with a similar method, as described by Fogle et al. (2005) and included DR and TR contributions. Resonances with the Rydberg electron in states with $n<30$ were considered explicitly, while recombination into selected states between $30<n<1000$ were calculated using a quantum defect approximation (Badnell et al. 2003). Recombination above $n=1000$ was considered insignificant and was not included in the calculations. Spectra including recombination into states up to $n=1000$ is hereafter termed as field-ionization-free data.

In the calculation, the energies for the $\Delta n=0$ core excitations were adjusted to match spectroscopic values from the NIST atomic database (Ralchenko et al. 2008), while for the $\Delta n=1$ excitation calculated energy values were used. The AUTOSTRUCTURE calculation contained radiative stabilization channels of the intermediate doubly excited state through decay of both the excited core and the Rydberg electron and autoionization rates to the original as well as to all other energetically available states have been considered. The DR contribution through the $2 \mathrm{~s} 3 l 3 l$ doubly excited states, i.e. both excitation and capture to $n=3$ shell, was considered in a separate calculation.

For comparison with the experiment, calculated rate coefficients were constructed by integrating the product of the 
calculated cross sections $(\sigma(v))$, velocity $(v)$, and $f\left(\mathrm{v}_{\mathrm{e}}\right)$ over the velocity space

$\alpha(E)=\int_{-\infty}^{+\infty} \sigma(v) v f\left(v_{\mathrm{e}}\right) \mathrm{d} v_{\mathrm{e}} \quad\left[\mathrm{cm}^{3} \mathrm{~s}^{-1}\right]$,

where $f\left(v_{\mathrm{e}}\right)$ is the electron velocity distribution from the experiment.

\section{Results and discussion}

\subsection{Merged-beam recombination rate coefficients}

Figure 1 shows the recombination spectrum of Be-like $\mathrm{Ne}$, up to $27.6 \mathrm{eV}$ energy. Two main series of peaks are present in this range, associated with excitation of the $2 \mathrm{~s}^{2}$ core $^{1}$ to the $2 \mathrm{~s} 2 \mathrm{p}\left({ }^{3} \mathrm{P}_{\mathrm{J}}\right)$ and $2 \mathrm{~s} 2 \mathrm{p}\left({ }^{1} \mathrm{P}_{1}\right)$ states. The recombination spectrum in this range also contains significant TR contributions (i.e. core excitation to the $2 \mathrm{p}^{2}$ configuration).

The energy balance of the resonant recombination channels is:

$E_{\mathrm{e}}=\Delta E_{\text {core }}-R y \frac{Q^{2}}{n^{2}}$,

where $E_{\mathrm{e}}$ is the kinetic energy of the free electron and $\Delta E_{\text {core }}$ is the excitation energy of the core. The last part in Eq. (4) gives the binding energy of the outer electron, with $R y$ and $Q$ being the Rydberg constant and charge number of the ion, respectively.

As the kinetic energy of the free electron increases, for the same core excitation, the outer electron will be attached into progressively higher $n l$ Rydberg states. The corresponding DR peaks are positioned closer to each other, forming a pile-up structure close to the series limits, well illustrated by DR resonances belonging to the $2 \mathrm{~s} 2 \mathrm{p}\left({ }^{1} \mathrm{P}_{1}\right) n l$ series.

As mentioned in Sect.2, field-ionization sets an upper limit on the detectable recombined states. However, ions recombined into states above $n_{\text {cutoff }}$ can decay to lower states during the $44.5 \mathrm{~ns}$ flight time between the electron cooler and the analyzing dipole magnet, and therefore can survive the passage through the magnet without being field-ionized. These ions are mostly responsible for the contribution extending above the field-ionization limit of the $2 \mathrm{~s} 2 \mathrm{p}\left({ }^{1} \mathrm{P}_{1}\right) n l$ series in the experimental rate coefficients.

In contrast to the $2 \mathrm{~s} 2 \mathrm{p}\left({ }^{1} \mathrm{P}_{1}\right) n l$ series, the $2 \mathrm{~s} 2 \mathrm{p}\left({ }^{3} \mathrm{P}_{\mathrm{J}}\right) n l$ type DR resonances are decreasing in strength toward higher energies, without creating an observable pile-up structure at the corresponding series limits. DR through ${ }^{3} \mathrm{P}_{\mathrm{J}}$ doubly excited states involves a spin-flip of one of the core electrons, to create a triplet excited core. Radiative decay of the ${ }^{3} \mathrm{P}_{\mathrm{J}}$ core is dipoleforbidden and thus radiative stabilization of the doubly excited state must take place through the decay of the Rydberg electron. The probability that the Rydberg electrons decay via radiative cascades below the ionization limit, before the doubly excited state has decayed by auto-ionization, rapidly decreases for increasing $n l$ states. Consequently, doubly excited states with a triplet excited core and high Rydberg electrons will mostly reautoionize, resulting in vanishing DR resonance strengths toward the ${ }^{3} \mathrm{P}_{\mathrm{J}}$ series limits.

RR into ground-state and into metastable ions has approximately the same value, thus all circulating ions recombined through this channel with the same rate. Hence, renormalization of the RR rate coefficients to account for the metastable content

\footnotetext{
1 The closed He-like core is ignored in the notation of the electron configurations.
}

in the ion beam was not necessary, when comparing calculated and experimentally derived results. The RR contribution to the measured recombination spectrum was estimated using Kramers formula (Kramers 1923)

$\sigma_{n}(E)=2.105 \times 10^{-22} g_{n} \frac{R y^{2} \cdot Z_{\mathrm{eff}}^{4}}{n E\left(n^{2} E+R y \cdot Z_{\mathrm{eff}}^{2}\right)}\left[\mathrm{cm}^{2}\right]$,

where $R y$ is the reduced mass Rydberg constant, $E$ is the centerof-mass energy, $n$ is the principal quantum number of the recombined electron, and $Z_{\mathrm{eff}}=8$ is the effective charge of the ion. The total RR cross section was obtained by summing over all available $n$ states, up to the field-ionization limit. This formula gives accurate cross sections for recombination into high $n$ states, as found also in a quantum mechanical description by Bethe \& Salpeter (1957). Toward lower $n$ states, the discrepancy between classical and quantum-mechanical description increases and Eq. (5) needs to be corrected by the Gaunt factor $g_{n}$ (Seaton 1959). The RR cross section was convoluted according to Eq. (3) to obtain RR rate coefficients.

For a valid comparison with the experimentally determined recombination spectrum, the AUTOSTRUCTURE ground-state rate coefficients (DR + TR) were scaled to $86 \%$, to account for the metastable fraction of the stored ion beam (see end of Sect. 4.1), and were added to the calculated RR rate coefficients. The resulting calculated $n_{\text {cutoff }}$ rate coefficients are shown in Fig. 1 by the solid (red) curve. Strong TR contributions were observed in the recombination spectrum over this energy range, associated with double excitation of the Be-like Ne core during the attachment of the free electron. The calculated trielectronic contribution to the spectrum (scaled to $86 \%$ ) is shown by the solid (black) curve, with the designation of the calculated resonances being written above the corresponding vertical arrows. Although the position of the TR resonances predicted by the calculation matches peaks in the experimentally derived spectrum, the strength of these peaks is significantly underestimated by the calculation. This is especially noticeable in case of the isolated peaks at $\sim 18 \mathrm{eV}$ and $20 \mathrm{eV}$, arising from recombination through the $2 \mathrm{p}^{2}\left({ }^{1} \mathrm{~S}_{0}\right) 4 l$ and $2 \mathrm{p}^{2}\left({ }^{1} \mathrm{D}_{2}\right) 5 l$ triply excited states, respectively. Similar discrepancies between calculated and experimental TR rates were observed in recombination spectra of other Be-like ions, e.g. for Be-like $\mathrm{Cl}$ (Schnell et al. 2003), and Be-like $\mathrm{Fe}$ (Savin et al. 2006).

At low-energies, below $450 \mathrm{meV}$, the recombination spectrum is dominated by a group of very strong, spin-forbidden resonances. Figure 2 shows a comparison between the experimentally determined rate coefficient spectrum and results of the AUTOSTRUCTURE calculations for ground-state ions summed with the calculated RR rate coefficients. Calculated resonance designations are given above the corresponding peaks. The calculation attributes all these peaks to DR through spinforbidden intermediate doubly excited states of the $2 \mathrm{~s} 2 \mathrm{p}\left({ }^{3} \mathrm{P}\right) 6 \mathrm{~d}$ and $2 \mathrm{~s} 2 \mathrm{p}\left({ }^{3} \mathrm{P}\right) 6 \mathrm{f}$ type. Although the number of peaks and the shape of the spectrum are similar, the calculated peaks are shifted toward higher energies and the calculated recombination strength between $1 \mathrm{meV}$ and $600 \mathrm{meV}$, is $\sim 32 \%$ lower than the experiment. Considering difficulties associated with calculation of DR resonances positioned close to threshold, the agreement between calculated and experimental results at low energies is remarkably good.

The recombination spectrum in the region of DR resonances associated with the excitation of a core electron to the $n>2$ shell is shown in Fig. 3. The energy scale of the calculation was stretched by a factor of 1.017 , to match experimental peak 


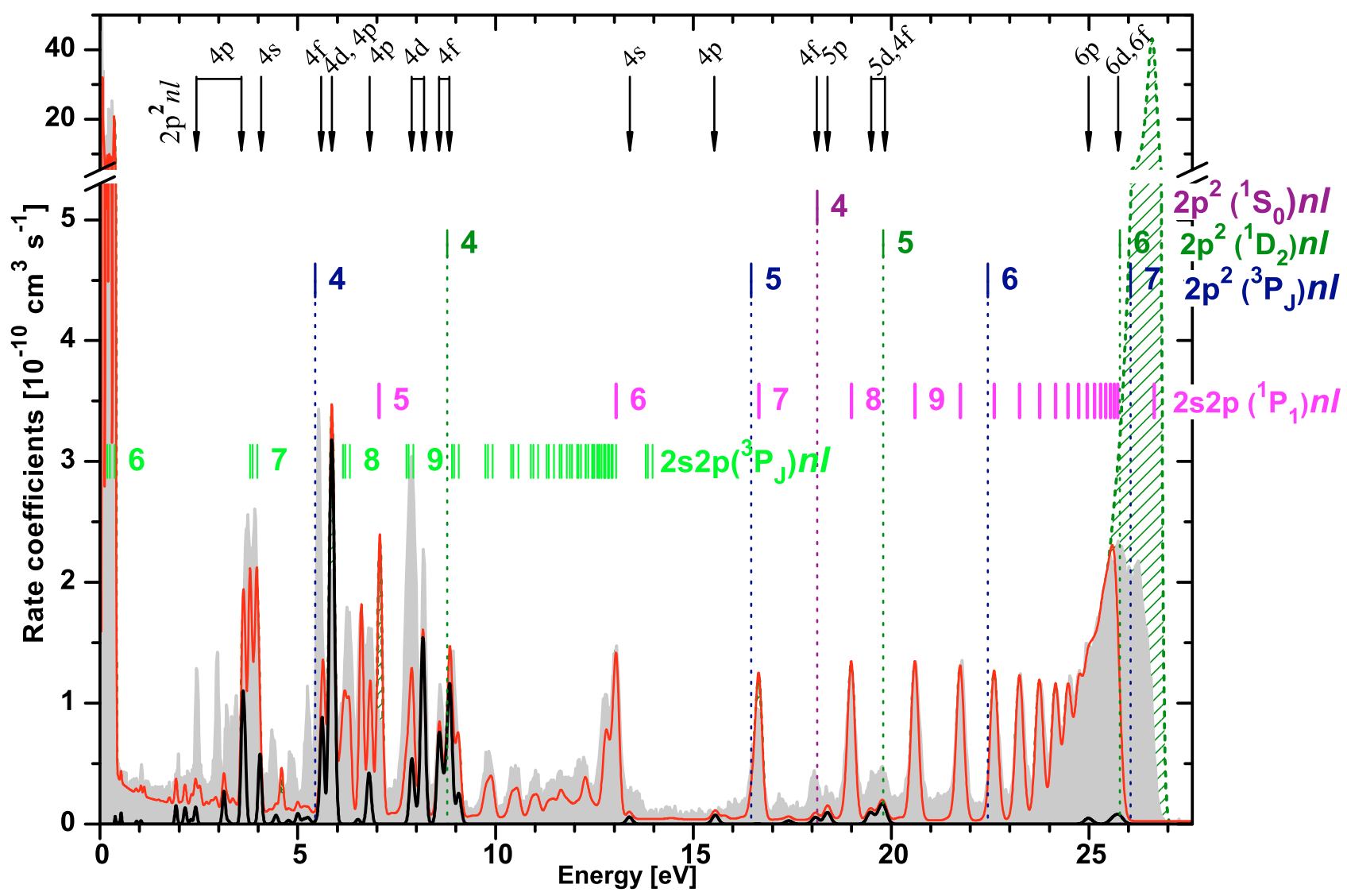

Fig. 1. Recombination rate coefficients of Be-like Ne, up to the $2 \mathrm{~s} 2 \mathrm{p}\left({ }^{1} \mathrm{P}_{1}\right) n l$ series limit. The gray area shows experimentally determined rate coefficients, arising mostly from the $86 \%$ ground state fraction of the stored ion beam. The red solid curve shows calculated ground-state recombination rate coefficients (RR + DR + TR) and the black solid curve shows calculated TR rate coefficients, both scaled to $86 \%$, as discussed in the text, to account for the metastable fraction of the ion beam present in the experiment. The hatched area shows the calculated field-ionization-free ground-state recombination rate coefficients, scaled to $86 \%$. Vertical bars show resonance positions calculated using Eq. (4), with the principal quantum number of the Rydberg electron written next to the bars. Vertical arrows show TR resonance positions and designations, extracted from the AUTOSTRUCTURE calculation.
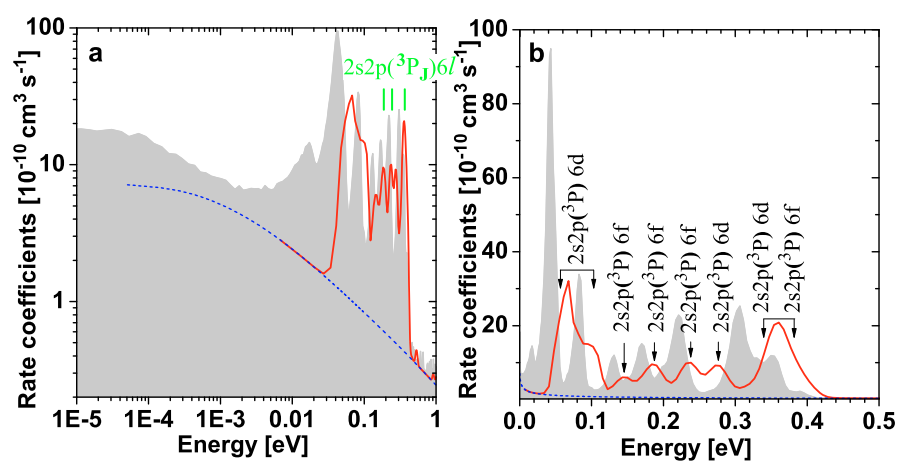

Fig. 2. Low-energy recombination rate coefficients of $\mathrm{Ne}^{6+}$. a) The gray area shows the experimentally derived rate coefficient spectrum. Contributions from RR are shown by the dashed line. The solid curve shows ground-state AUTOSTRUCTURE rate coefficients scaled to $86 \%$ and summed with the RR rate coefficients. Resonance positions estimated with Eq. (4) are shown by the vertical bars. b) Same plot designations as in part a) of the figure. The vertical arrows and associated labels show resonance positions and designations in the AUTOSTRUCTURE calculation.

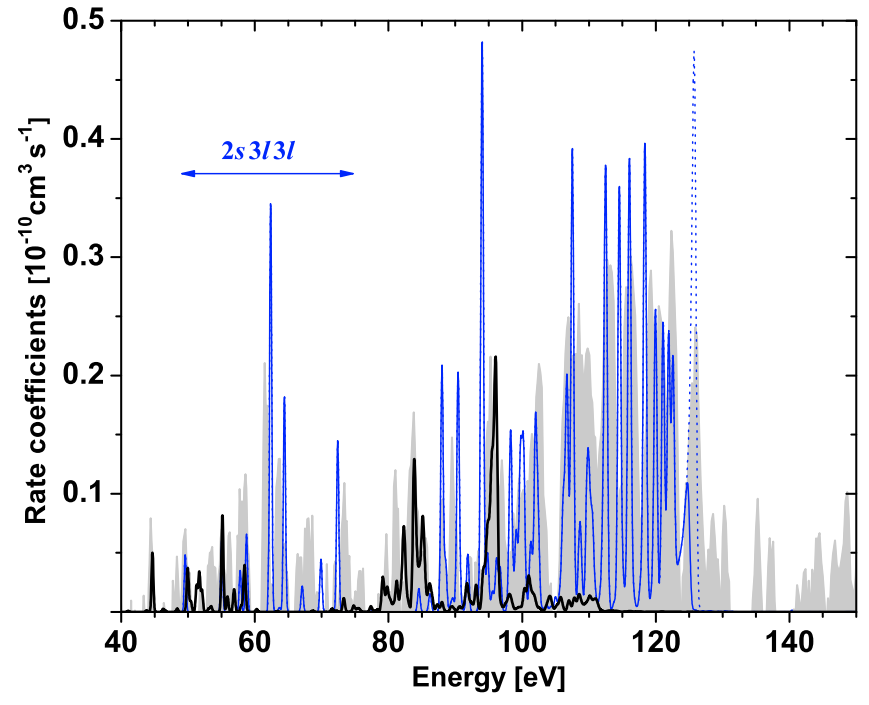

Fig. 3. Recombination rate coefficients of Be-like Ne in the $\Delta n=1$ region. The gray area shows the experimentally determined rate coefficient spectrum. Solid blue curve shows calculated ground-state recombination rate coefficients scaled to $86 \%$ and the solid black curve shows the calculated metastable rate coefficients scaled to $14 \%$. The dotted curve shows the ground-state calculations that include recombination into states up to $n=1000$. 
positions. In the figure, the energy range of DR through the $2 \mathrm{~s} 3 l 3 l$ doubly excited states is shown by the horizontal arrow.

The ground state of Be-like ions, $2 \mathrm{~s}^{2}\left({ }^{1} \mathrm{~S}_{0}\right)$, consists of two electrons with antiparallel spins. Metastable ions with very long lifetimes are associated with the $2 \mathrm{~s} 2 \mathrm{p}\left({ }^{3} \mathrm{P}_{\mathrm{J}}\right)$ states in this isoelectronic sequence (Marques et al. 1993). In the absence of a nuclear spin, the lifetime of the ${ }^{3} \mathrm{P}_{0}$ state is essentially $\infty$ (Brage et al. 1998; Cheng et al. 2008), whereas the other ${ }^{3} \mathrm{P}_{\mathrm{J}}$ states are expected to decay during the acceleration and cooling of the ion beam.

Separate AUTOSTRUCTURE calculations provided cross sections for ground-state and ${ }^{3} \mathrm{P}_{0}$ metastable-state ions recombining with free electrons. These metastable ions can survive transportation and acceleration in the storage ring and can be present in the ion beam during our measurements. In the range of the $\Delta n=0$ DR resonances, the calculation did not show any significant metastable DR strength. In the $\Delta n=1$ region, on the other hand, distinct contributions from metastable ions were predicted by the calculation (see Fig. 3). A 14\% metastable content in the stored ion beam was determined by scaling the calculated ground-state and metastable rate coefficients to match the level of the measured recombination spectrum. Calculated contributions to the spectrum, arising from recombination into states located above the experimental field-ionization limit are shown in Fig. 3 by the dotted curve.

The $14 \%{ }^{3} \mathrm{P}_{0}$ metastable fraction, estimated by comparing calculated and experimentally derived rate coefficient spectra, is supported by other experiments with Be-like ions. Previously, ion beams extracted from the same ion source as used in this experiment, showed decreasing metastable content with increasing charge, along the Be-like isoelectronic sequence. In those experiments, metastable contents of $60 \%, 40 \%$, and $35 \%$ were found for $\mathrm{C}^{2+}, \mathrm{N}^{3+}$, and $\mathrm{O}^{4+}$ ion beams, respectively (Fogle et al. 2005). In another experiment, similar metastable fractions were determined in Be-like $\mathrm{C}, \mathrm{N}$, and $\mathrm{O}$ ion beams extracted from an ECR ion source (Brazuk et al. 1984). The metastable content obtained here matches well the trend observed in the above experiments.

Assuming that the metastable fraction is accurate within $50 \%$ of the derived value, the uncertainty in the magnitude of the experimental rate coefficients, associated with the procedure used to determine the metastable fraction of the ion beam, ammounts to $\sim 7 \%$. The uncertainty (typical to DR experiments performed at storage rings) of $15 \%$, due to counting statistics, uncertainties in beam currents and interaction length, combined with the uncertainties in the metastable fraction of the ion-beam amounts to a total error of $\sim 17 \%$.

The experimentally determined spectra were converted to pure ground-state $\mathrm{Ne}^{6+}$ rate coefficients with the following procedure: first the metastable contribution was subtracted from the experimentally derived rate coefficient spectra, using the calculated metastable results. The resulting recombination spectra were then renormalized for $100 \%$ ground-state ion beam, by dividing the rate coefficients by 0.86 . The obtained pure groundstate merged-beam recombination rate coefficients were used to obtain ground-state plasma rate coefficients, as described in the following section.

\subsection{Plasma recombination rate coefficients}

In contrast with the narrow energy distribution of the electron beam in our experiment, in a plasma, ions are colliding with

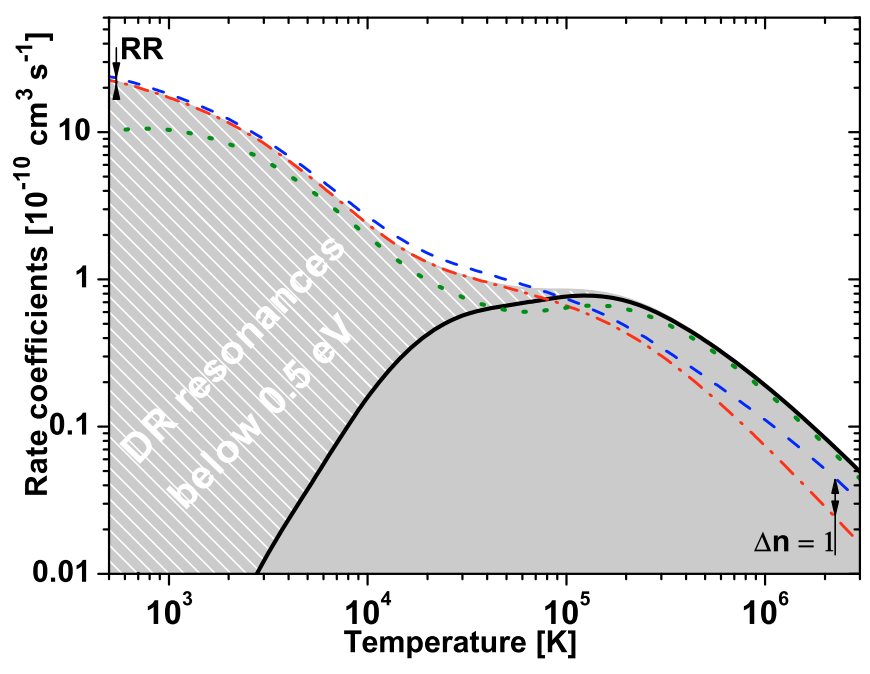

Fig. 4. Plasma recombination rate coefficients of Be-like Ne. The red dash-dotted line shows the RR subtracted $\Delta n=0$ plasma recombination rate coefficients. The blue dashed curve shows the total $n_{\text {cutoff }}$ plasma rate coefficients derived from the experiment. Plasma rate coefficients derived from field-ionization-free AUTOSTRUCTURE results are shown by green dots. Experimentally derived field-ionization-free DR + TR rate coefficients are shown by the gray area. Contributions arising from the low-energy spin-forbidden DR resonances are emphasized by the hatched area.

electrons having a wide range of energies, described by the Maxwell-Boltzmann energy distribution

$f\left(E, T_{\mathrm{e}}\right)=\frac{2 E^{1 / 2}}{\pi^{1 / 2}\left(k T_{\mathrm{e}}\right)^{3 / 2}} \exp \left(-\frac{E}{k T_{\mathrm{e}}}\right)$,

where $E$ is the electron energy, $T_{\mathrm{e}}$ is the plasma temperature and $k$ is the Boltzmann constant. Recombination then takes place through a large number of DR channels, with strengths determined by the electron energy distribution function. To obtain plasma recombination rate coefficients, $\alpha\left(T_{\mathrm{e}}\right)$, as a function of plasma temperature $T_{\mathrm{e}}$, the merged-beam rate coefficient spectra were convoluted with energy distribution functions specific to the corresponding temperatures

$\alpha\left(T_{\mathrm{e}}\right)=\int \alpha(E) f\left(E, T_{\mathrm{e}}\right) \mathrm{d} E$,

where $\alpha(E)$ is the merged-beam recombination rate coefficient spectra. This approach is valid, as long as the electron energy spread in the merged-beam experiment is much narrower than the electron energy spread in the plasma, i.e. the merged-beam spectrum is a good approximation for the recombination cross section. The principles for deriving plasma rate coefficients from storage-ring measurements have been laid out by Savin (1999). Later, Schippers et al. (2001) examined the procedure in detail, with emphasis on the effect of the finite experimental energy spread on the low-temperature plasma rate coefficients.

The pure ground-state merged-beam recombination rate coefficients were used to obtain plasma rate coefficients shown in Fig. 4 by the dashed line. The dash-dotted line in Fig. 4 gives the $\Delta n=0$ resonant contribution to the plasma rate coefficients, i.e. $\mathrm{DR}+\mathrm{TR}$ up to the $2 \mathrm{~s} 2 \mathrm{p}\left({ }^{1} \mathrm{P}_{1}\right) n l$ series-limit. The difference between the dashed and dash-dotted curves at low temperatures originates from the experimentally measured RR, whereas at high temperatures recombination associated with excitation of the core electrons to $n>2$ states becomes important. 
Peaks belonging to the $2 \mathrm{~s} 2 \mathrm{p}\left({ }^{3} \mathrm{P}_{\mathrm{J}}\right) n l$ series are gradually decreasing toward $n=\infty$ and the field-ionization-free AUTOSTRUCTURE calculation did not show significant recombination strength into states above the experimental fieldionization limit. At the $2 \mathrm{~s} 2 \mathrm{p}\left({ }^{1} \mathrm{P}_{1}\right) n l$ series limit on the other hand, a substantial amount of the recombined ions were fieldionized in the experiment, as emphasized by the hatched area in Fig. 1. To account for the fraction of recombined ions fieldionized in the experiment, results of the field-ionization-free AUTOSTRUCTURE calculation were used between 25.5 and $27.2 \mathrm{eV}$. A similar procedure was applied to the experimentally derived merged-beam recombination spectrum at higher energies, where the metastable contribution was first subtracted from the experiment and then AUTOSTRUCTURE results were used at energies where the calculation predicted field-ionization of recombined ions. The resulting merged-beam rate coefficients accounting for field-ionization were then convoluted to obtain plasma rate coefficients, shown in Fig. 4 by the gray area, hereafter termed as field-ionization-free plasma rate coefficients.

Similarly to the Be-like $\mathrm{Ne}$ case, in the recombination spectra of other Be-like ions, $\mathrm{Mg}^{8+}, \mathrm{O}^{4+}$, strong low-energy resonances are overwhelmingly contributing to the plasma recombination rate coefficients (Schippers et al. 2004; Fogle et al. 2005). Accurate position of low-energy resonances is essential for the accuracy of the derived plasma rate coefficients. Even small shifts in the position of low-energy resonances have drastic effects on the magnitude of plasma rate coefficients at low temperatures. These uncertainties are especially important for low-Z ions within a certain isoelectronic sequence, because these ions are abundant at temperatures where uncertainties in the position of low-energy resonances have large effects on the plasma recombination rate coefficients.

To investigate the impact on the $\mathrm{Ne}^{6+}$ plasma rate coefficients, arising from the low-energy spin-forbidden resonances, the merged-beam recombination spectrum below $500 \mathrm{meV}$ was removed and the remaining part was used to convolute plasma recombination rate coefficients. The obtained rate coefficients are shown in Fig. 4 by the thick solid curve, the hatched area emphasizing the contribution from the low-energy spin-forbidden resonance group. In plasmas with temperatures up to $10^{4} \mathrm{~K}$, recombination of Be-like $\mathrm{Ne}$ takes place almost exclusively through ${ }^{3} \mathrm{P}_{\mathrm{J}}$ core-excited states. Emission lines originating from the decay of the ${ }^{3} \mathrm{P}_{\mathrm{J}}$ excited states, efficiently populated by DR at such temperatures, could provide a useful diagnostic tool for astrophysics.

The resonant plasma rate coefficients were fitted with an expression similar to the formula originally suggested by Burgess (1965) for the description of DR in plasma,

$\alpha\left(T_{\mathrm{e}}\right)=T_{\mathrm{e}}^{-3 / 2} \sum_{i} c_{i} \cdot \exp \left(-\frac{E_{i}}{k T_{\mathrm{e}}}\right)$,

where $c_{i}$ and $E_{i}$ are fit parameters. The coefficients obtained from the fitting procedures are shown in Table 1 , and are accurate within $0.2 \%$ up to $3 \times 10^{5} \mathrm{~K}$ and better $\sim 0.6 \%$ up to $10^{6} \mathrm{~K}$.

\section{Comparison with data from literature}

Our field-ionization-free resonant (i.e. DR + TR) plasma rate coefficients are compared in Fig. 5 with data available in the literature. Resonant recombination channels dominate over RR in the entire investigated temperature range, and at $10^{3} \mathrm{~K}$ are more than one order of magnitude higher than the RR data of Pequignot et al. (1991).
Table 1. Fit coefficients for the RR-subtracted $n_{\text {cutoff }}$ and RR-subtracted field-ionization-free plasma rate coefficients from Fig. 4 . The dimensions of $c_{i}$ and $E_{i}$ are $10^{-5} \mathrm{~cm}^{3} \mathrm{~s}^{-1} \mathrm{~K}^{1.5}$ and $\mathrm{eV}$, respectively.

\begin{tabular}{rrrrr}
\hline \hline$\#$ & $n_{\text {cutoff }}$ & \multicolumn{3}{c}{$n=1000$} \\
$i$ & $c_{i}$ & $E_{i}$ & $c_{i}$ & $E_{i}$ \\
\hline 1 & 6.57 & 0.044 & 6.653 & 0.044 \\
2 & 11.68 & 0.186 & 12.23 & 0.191 \\
3 & 10.30 & 0.360 & 9.729 & 0.369 \\
4 & 39.77 & 2.950 & 45.64 & 3.063 \\
5 & 222.30 & 6.761 & 235.33 & 7.108 \\
6 & 579.80 & 20.685 & 1821.70 & 24.473 \\
7 & 852.30 & 102.80 & 915.00 & 100.820 \\
\hline
\end{tabular}

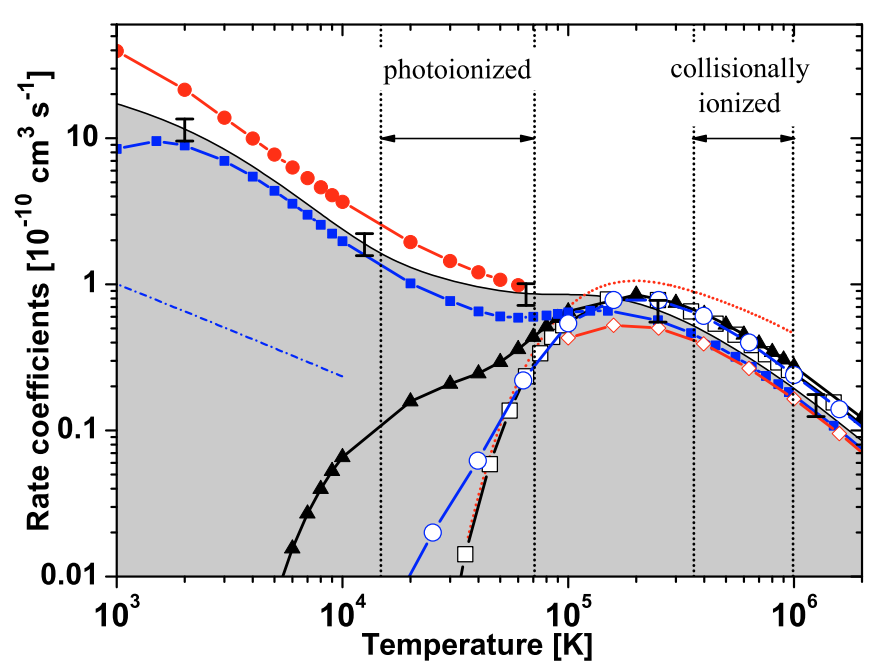

Fig. 5. Comparison of field-ionization-free resonant plasma recombination rate coefficients shown by gray area, with calculated data from the literature. Error bars show $17 \%$ uncertainty in our rate coefficients. Full circles and full squares show rate coefficients by Nussbaumer \& Storey (1987) and Colgan et al. (2003), respectively. Calculations by Romanik (1988) and Jacobs et al. (1979) are shown by full triangles and open circles, respectively. Rate coefficients from a compilation by Shull \& van Steenberg (1982) are shown by open squares. Results of Aldrovandi \& Pequignot (1973) are given by dotted line and the data of Badnell (1987) is marked by open diamonds. The only RR plasma rate coefficients in the figure, by Pequignot et al. (1991), are shown by dash-dotted line. Temperature ranges in photoionized and collisionally ionized plasma, where the Be-like Ne concentration is higher than $10 \%$ of its maximum abundance are shown by horizontal arrows (Kallman \& Bautista 2001).

Temperature intervals in photoionized and collisionallyionized plasma, where the concentration of Be-like $\mathrm{Ne}$ is higher than $10 \%$ of the maximum abundance, are shown by horizontal arrows (Kallman \& Bautista 2001). In the temperature region where Be-like $\mathrm{Ne}$ is expected to be abundant in photoionized plasma, discrepancies of several orders of magnitude can be observed between the results of different calculations. Most calculations yield vanishing plasma rate coefficients at low temperatures, with only two theoretical results having comparable values to our rate coefficients below $5 \times 10^{4} \mathrm{~K}$.

Nussbaumer \& Storey (1987) evaluated low-temperature DR plasma recombination rate coefficients by calculating transition rates within the frame of LS coupling. The spin-orbit interaction was then considered strong enough to allow LS forbidden autoionization of some doubly excited states located above the $2 s^{2} n l$ ionization threshold of the recombined ion. This approach showed a factor of 3.8 increase in the plasma recombination rate 
coefficients at $10^{4} \mathrm{~K}$, as compared to the purely LS allowed case. These results are higher than our rate coefficients over the entire presented range.

For temperatures below $3 \times 10^{4} \mathrm{~K}$, including the temperature range where Be-like $\mathrm{Ne}$ is abundant in photoionized plasma, our results are closest to the rate coefficients calculated from fit coefficients given by Colgan et al. (2003).

Mazzotta et al. (1998) give a compilation of plasma rate coefficients for all elements between $\mathrm{H}$ and Ni. For Be-like $\mathrm{Ne}$ they use calculated results by Badnell (1987), shown in Fig. 5 by open diamonds.

Shull \& van Steenberg (1982) give a compilation of fit coefficients for plasma rate coefficients of astrophysically important ions. For Be-like Ne calculations of Jacobs et al. (1979) were fitted, however the fit coefficients given by Shull \& van Steenberg (1982) reproduce accurately only the range above $5 \times 10^{4} \mathrm{~K}$, deviating significantly from the original data below this temperature.

Be-like $\mathrm{Ne}$ is predicted by Jacobs et al. (1979) to reach maximum abundance at temperatures of $\sim 5 \times 10^{5} \mathrm{~K}$, matching temperatures where Kallman \& Bautista (2001) predict high abundances of this ion in collisionally ionized plasma. In their calculation, Jacobs et al. (1979) consider primarily recombination channels with the outer electron in highly excited $n$ states. The recombination rate coefficients are rapidly diverging from our results for temperatures lower than $10^{5} \mathrm{~K}$. We note that in their publication, the plasma rate coefficient value at $10^{4.6} \mathrm{~K}$ seems to be mistyped and is one order of magnitude higher.

Above $3 \times 10^{5} \mathrm{~K}$, the calculated results of Badnell (1987) and Colgan et al. (2003) are in agreement (within 17\%) with our results. The calculations of Jacobs et al. (1979) and Romanik (1988) have similar values and are $\sim 30 \%$ higher than our results. The rate coefficients calculated by Aldrovandi \& Pequignot (1973) in this range are significantly higher than the other calculations.

At even higher temperatures, DR through inner shell excitation of the Be-like core (i.e., capture of a free electron with the simultaneous excitation of a 1s electron to a higher available state) can become important. Our merged-beam experiment did not probe such high electron-ion collision energies, where this type of process occurs, thus our data does not include contributions from these recombination channels. However, Jacobs et al. (1979) estimate this contributions to become sizable at temperatures higher than $3 \times 10^{6} \mathrm{~K}$, which is above the range considered here.

\section{Conclusions}

Recombination rate coefficients of Be-like $\mathrm{Ne}$ recombining into $\mathrm{B}$-like $\mathrm{Ne}$ were derived for the first time from a measurement performed at CRYRING heavy-ion storage ring. Long lived metastable Be-like ions were present in the ion beam during the experiment. The AUTOSTRUCTURE calculation predicted metastable contributions to the recombination spectrum at energies where $\Delta n=1 \mathrm{DR}$ takes place. A $14 \%$ fraction of metastable ions was estimated from the comparison of the experimentally obtained spectrum, with calculated rate coefficients for pure ground and for pure metastable initial ions.

Good agreement was found between experimentally derived rate coefficients and results of AUTOSTRUCTURE calculations. In the energy range of $\Delta n=0$ DR resonances, strong TR contributions were found in the recombination spectrum. The TR resonance positions were well predicted by the
AUTOSTRUCTURE calculation, however the calculation underestimated the strength of the associated peaks.

Recombination through spin-forbidden $2 \mathrm{~s} 2 \mathrm{p}\left({ }^{3} \mathrm{P}\right) 6 \mathrm{~d}$ and $2 \mathrm{~s} 2 \mathrm{p}\left({ }^{3} \mathrm{P}\right) 6 \mathrm{f}$ doubly excited states form a group of very large resonances at energies below $450 \mathrm{meV}$. These resonances have a dramatic effect on the plasma recombination rate coefficients at low temperatures. At $10^{3} \mathrm{~K}$, recombination through these resonances is more than one order of magnitude larger than through the nonresonant RR channel.

Below $10^{5} \mathrm{~K}$, including the region where Be-like $\mathrm{Ne}$ is expected to be abundant in photoionized plasma, large differences appear between the results of various calculations, with only two calculations from the literature showing rate coefficients comparable to our experimental results.

At higher temperatures, above $2 \times 10^{5} \mathrm{~K}$, better agreement exists between the results of different calculations. Here, our results are closest to calculated results of Colgan et al. (2003) and Badnell (1987).

Acknowledgements. We acknowledge the excellent support of the CRYRING crew during the experiment. S.D.L. would like to acknowledge the generous support of the Oak Ridge National Laboratory for funding part of this work.

\section{References}

Abrahamsson, K., Andler, G., Bagge, L., et al. 1993, NIMPRB, 79, 269

Aldrovandi, S. M. V., \& Pequignot, D. 1973, A\&A, 25, 137

Anders, E., \& Grevesse, N. 1989, Geochim. Cosmochim. Acta, 53, 197

Badnell, N. R. 1986, J. Phys. B: Atom. Molec. Phys., 19, 3827

Badnell, N. R. 1987, J. Phys. B: Atom. Molec. Phys., 20, 2081

Badnell, N. R., O'Mullane, M. G., Summers, H. P., et al. 2003, A\&A, 406, 1151

Bethe, H., \& Salpeter, E. 1957, The quantum mechanics of one- and twoelectron systems, Handbuch der Physik (Berlin: Springer), 35

Brage, T., Judge, P. G., Aboussaid, A., et al. 1998, ApJ, 500, 507

Brazuk, A., Dijkkamps, D., Drentje, A. G., de Heer, F. J., \& Winter, H. 1984, J. Phys. B: Atom. Molec. Phys., 17, 2489

Burgess, A. 1964, ApJ, 139, 776

Burgess, A. 1965, ApJ, 141, 1588

Cheng, K. T., Chen, M. H., \& Johnson, W. R. 2008, Phys. Rev. A, Atom., Molec., and Optic. Phys., 77, 052504

Colgan, J., Pindzola, M. S., Whiteford, A. D., \& Badnell, N. R. 2003, A\&A, 412, 597

Danared, H., Källberg, A., Andler, G., et al. 2000, NIMPRA, 441, 123

Fogle, M., Badnell, N. R., Glans, P., et al. 2005, A\&A, 442, 757

Jacobs, V. L., Davis, J., Rogerson, J. E., \& Blaha, M. 1979, ApJ, 230, 627

Kallman, T., \& Bautista, M. 2001, ApJS, 133, 221

Kramers, H. A. 1923, Philos. Mag., 46, 836

Marques, J. P., Parente, F., \& Indelicato, P. 1993, Phys. Rev. A, 47, 929

Mazzotta, P., Mazzitelli, G., Colafrancesco, S., \& Vittorio, N. 1998, A\&AS, 133, 403

Nussbaumer, H., \& Storey, P. J. 1987, A\&AS, 69, 123

Orban, I., Lindroth, E., Glans, P., \& Schuch, R. 2007, J. Phys. B: Atom., Molec. and Optic. Phys., 40, 1063

Pequignot, D., Petitjean, P., \& Boisson, C. 1991, A\&A, 251, 680

Poth, H. 1990, Phys. Rep., 196, 135

Ralchenko, Y., Kramida, A., Reader, J., \& NIST ASD Team. 2008, NIST Atomic Spectra Database, version 3.1.4, National Institute of Standards and Technology, Gaithersburg, MD, http://physics.nist.gov/asd3 Romanik, C. J. 1988, ApJ, 330, 1022

Savin, D. W. 1999, ApJ, 523, 855

Savin, D. W., Gwinner, G., Grieser, M., et al. 2006, ApJ, 642, 1275

Schippers, S., Müller, A., Gwinner, G., et al. 2001, ApJ, 555, 1027

Schippers, S., Schnell, M., Brandau, C., et al. 2004, A\&A, 421, 1185

Schippers, S., Schmidt, E. W., Bernhardt, D., et al. 2007, J. Phys. Conf. Ser., 58, 137

Schnell, M., Gwinner, G., Badnell, N. R., et al. 2003, Phys. Rev. Lett., 91, 043001

Seaton, M. J. 1959, MNRAS, 119, 81

Shull, J. M., \& van Steenberg, M. 1982, ApJS, 48, 95 